\title{
GER-E-TECH: Telemonitoring Project for Elderly Residents in Nursing Homes
}

\author{
Zulfiqar Abrar-Ahmad*1, Kadri Nadir' ${ }^{1}$, Doucet Jean', Hajjam Amir ${ }^{2}$, Talha Samy ${ }^{3}$, Hajjam Mohamed ${ }^{4}$, \\ Hajjam Jawad ${ }^{5}$, Ervé Sylvie ${ }^{5}$, Gény Bernard ${ }^{6}$, Letourneau Dominique ${ }^{7}$ and Andrès Emmanuel ${ }^{8}$
}

${ }^{1}$ Department of Geriatrics-Therapeutics, Saint Julien Hospital, Rouen, France

${ }^{2}$ Research Unit EA 4662 Nanomedicine, Imaging, Therapeutics, Belfort-Montbéliard, France

${ }^{3}$ Research Unit EA 3072 Mitochondrie, Stress oxydant et Protection muscular, Strasbourg, France

${ }^{4}$ PREDIMED Technology, Mulhouse, France

${ }^{5}$ Expert Center for Information and Communication Technologies for Autonomy, Angers, France

${ }^{6}$ Department of Physiology and Functional Explorations, Strasbourg, France

${ }^{7}$ Foundation of the Future for Applied Medical Research, Paris, France

${ }^{8}$ Department of Internal Medicine, Diabetes, Strasbourg, France

*Corresponding author: Zulfiqar Abrar-Ahmad, Department of Geriatrics-Therapeutics, University Hospital of Rouen, France

\section{ARTICLE INFO}

Received: 崑 February 19, 2019

Published: 蔧 February 28, 2019

\section{ABSTRACT}

Citation: Zulfiqar AA, Kadri N, Doucet J, Hajjam A, Talha S, Hajjam M, Hajjam J, Ervé S, Gény $B$, Letourneau D, Andrès E. GER-E-TECH: Telemonitoring Project for Elderly Residents in Nursing Homes. Biomed J Sci \& Tech Res 15(2)-2019. BJSTR. MS.ID.002687.

\section{Introduction}

Elderly and very elderly people living in nursing homes are frequently being hospitalized, more often than not in emergency situations, and the number is steadily increasing. To combat this problem, the French government has implemented a policy to increase accommodation by building new nursing homes across most regions of France. Thus, the number of patients admitted to hospital emergency departments from nursing homes continues to grow year after year, particularly for people in nursing homes with little medical attention that have no night care nurses, and that often have very limited medical time (coordinating doctors). Nursing home healthcare staff must be given tools to limit the flow of these poly pathological older patients to Emergency Services such as assistance in the prevention of decompensation of some geriatric syndromes to create a preventive policy and improve quality of life for the residents. Literature data supports this: transfer to Emergency Units cause increased rates of morbidity and mortality.

\section{Telemedicine: Towards Preventive Medicine}

According to the Public Health Code, telemedicine is defined as a form of remote medical practice that uses information and communication technologies. It connects one or more health professionals to themselves or to a patient; this includes a medical professional and, where appropriate, other professionals providing patient care. It allows healthcare professionals, with or without their patient's presence, to connect with one another, or with specialists involved in the patient's care. Telemedicine is a recent development in healthcare, enabled by technological progress and promoted by public authorities due to its potential possibilities, including improved accessibility to care, optimized use of professionals' time, improved collaboration between professionals, optimized care pathways, and revived innovation in therapeutic treatment plans. Telemedicine has shown its effectiveness in the management of chronic diseases, such as heart failure and high blood pressure, among others. Monitoring patients with chronic diseases through 
the use of telemedicine is a way to optimize their care. It also offers a better quality of life for elderly patients. Telemonitoring could lead to a significant reduction in the number of readmissions, which would consequently result in lower costs for society. These solutions also encourage the accurate collection of medical data to enable improved monitoring of patient health. The objectives are thus ambitious, ranging from improved rates of morbidity and mortality, to decreased readmissions, improved quality of life and reduced economic costs.

\section{GER-E-TECH: Telemonitoring Project for Nursing Home}

With this in mind, we set up the Ger-e-Tech project to study the telemonitoring of residents in Rouen University Hospital nursing homes. Through the structuring and recording of medical care, we aim to avoid situations of acute decompensation and complications of geriatric risks. Our objective is to develop a codified, preventive approach for the management of major geriatric risks in nursing homes using a personalized telemonitoring platform dedicated to the residents, in order to avoid the occurrence of an acute decompensation factor in the elderly. The collection of information by the platform will not only allow personalized monitoring but will increase patient knowledge and provide a particularly effective tool for transmission between nursing staff (doctors, nurses, etc.) in nursing homes, thus allowing continuity of care. The collected data will also extract markers to improve early detection of any decompensation, thereby improving patient monitoring and reducing hospitalizations. Further, this work will allow for the creation of resident liaison files, providing any paramedical and medical health professional with the resident's up-to-date geriatric data, including anthropometric, nutritional, cognitive and iatrogenic data. Thanks to a simple and streamlined approached, this constitutes a real illustration integrated into the electronic platform of the standardized gerontological evaluation. Geriatric risks will include the risk of falling, constipation, dehydration, confusion, iatrogenicity, undernutrition, heart failure, diabetes, infections and bedsores.

The project's objective is to experiment with recorded personalized medical monitoring of Rouen University Hospital nursing home residents using the E-care intelligent telemedicine platform, winner of the Investissements d'Avenir (Future Investments) in 2012 and the Foundation d'Avenir (Future Foundation) in 2015 and 2017. The platform assists caregivers by automating the processing of information from sensors and questionnaires to detect anomalies and make early diagnoses of medically risky situations. E-care will provide personalized care for major geriatric risks to avoid the occurrence of an acute decompensation factor in elderly patients. The information collected will be supplemented by codified therapeutic management that follows international recommendations, for direct use in nursing homes. The project will rely on a multidisciplinary team built on the necessary medical, scientific and structural skills. The E-care platform uses an intelligent algorithm to process data and generate alerts based on medical knowledge of the pathologies treated and modeled by ontologies. The general principle adopted by this platform is the anticipation of decompensation through the detection of warning signs that ultimately lead to hospitalization. Tested at the Strasbourg University Hospital from 2013 to 2014 and in homes in 2015, the platform is currently deployed in the PRADOINCADO project in Strasbourg (a collaborative project between the Strasbourg University Hospital, the Bas-Rhin Medical Insurance and the Grand-Est Regional Health Agency) to monitor heart failure patients where they live. This platform is in the process of being certified as an EU Medical Device and the Diabetes platform has recently been accredited by the Alsace Bio Valley Competitiveness and Innovation Centre. We will develop a series of measures and questions to be integrated into the E-Care platform for the personalized and adaptive monitoring of patient health in nursing homes. This study, starting in September 2018, is supported by the CENTICH (Centre expertise National des Technologies de information et de la Communication pour autonomy, National Expertise Centre for Information Technologies and Communication for Autonomy) [1].

\section{References}

1. Andrès E, Talha S, Benyahia A, Keller O, Hajjam M, et al. (2016) Experimentation of an e-platform to detect situations at risk of cardiac impairment (platform E-care) in an internal medicine unit. Rev Med Interne 37(9): 587-593

\section{ISSN: 2574-1241}

DOI: 10.26717/BJSTR.2019.15.002687

Zulfiqar A A, et al. Biomed J Sci \& Tech Res

This work is licensed under Creative

Commons Attribution 4.0 License

Submission Link: https://biomedres.us/submit-manuscript.php

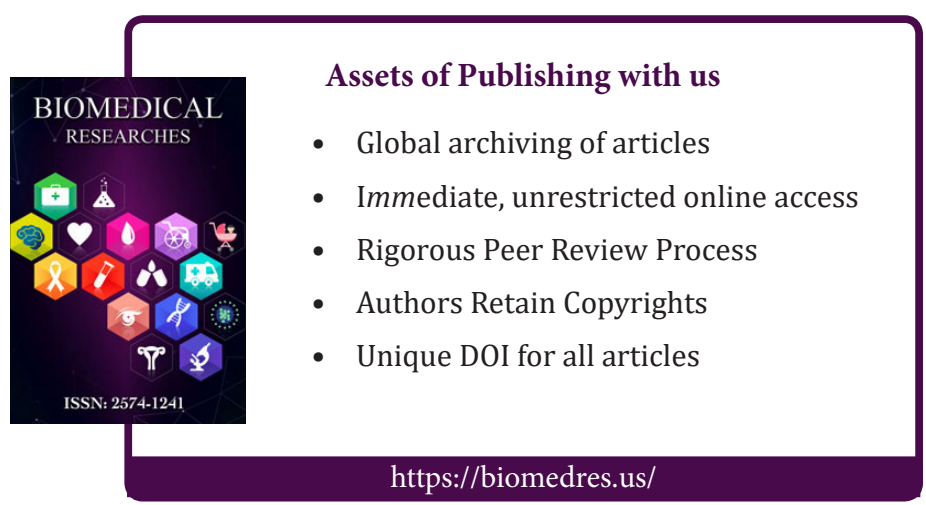

